\title{
Assessing final year pharmacy students' knowledge on cervical cancer and the NHS Cervical Screening Programme
}

Authors: Cara Cully, Rosalind Wyatt, Professor Kay Marshall, Professor Kaye Williams, Dr Rebecca Elvey. Manchester Pharmacy School, The University of Manchester

Background: It has been long established that infection with specific types of human papillomavirus (HPV) has been associated with cervical cancer. Since the introduction of the NHS Cervical Screening Programme in 1988, UK mortality rates of the disease have decreased by almost two thirds. Recent years have witnessed an increase in cervical cancer incidence rates. In addition, screening rates are failing to reach the government's target of $80 \%$.

Method: A questionnaire was designed and developed to assess final year pharmacy students' knowledge of HPV and cervical cancer screening. Students' opinions regarding their current level of knowledge and whether pharmacists should have a role in the public awareness of cervical screening were also assessed. Questionnaires were distributed to final year students $(n=159)$ during an attendancerecorded class.

Results: A total of 131/159 questionnaires were returned (response rate $82.4 \%$ ). Knowledge based on 13 multiple-choice questions varied greatly between individuals (Mean score $=6.15, \mathrm{SD}=1.97$ ). Background knowledge associated with HPV and cervical cancer was significantly greater than cervical screening knowledge $(P<0.001)$. Students who studied an optional module, Microbial Disease and Immunisation, had significantly higher levels of cervical screening knowledge than those students who did not $(P=0.031)$. Over $90 \%$ of respondents agreed that pharmacists should have a role in public awareness of cervical screening.

Discussion: Poor levels of cervical screening knowledge largely reflect deficits in direct teaching within the MPharm curriculum. This information is only provided by an optional module which is taught during the fourth year of study. Differences in performance between students who studied/did not study this module demonstrate its effectiveness in raising awareness of cervical screening among prospective pharmacists. Consideration should be given to the provision of this module's content to all final year students. 